\title{
Intuitionistic Fuzzy Modeling to Travelling Salesman Problem
}

Arindam Garai, Tapan Kumar Roy

Department of Mathematics, Sonarpur Mahavidyalaya, West Bengal, India, Pin: 700149

fuzzy_arindam@yahoo.com

Department of Mathematics, Bengal Engineering and Science University, Howrah, India, Pin: 711103

\section{ABSTRACT}

roy_t_k@yahoo.co.in

This paper presents solution technique for travelling salesman problem (TSP) under intuitionistic fuzzy environment. Travelling salesman problem is a non-deterministic polynomial-time (NP) hard problem in combinatorial optimization, studied in graph theory, operations research and theoretical computer science. It must be noted that a traveling sales man even face a situation in which he is not able to achieve his objectives completely. There must be a set of alternatives from which he can select one that best meets his aspiration level. For Multi-Objective Symmetric TSP, in fuzzy environment, it is converted into a Linear Program using Fuzzy Multi-Objective Linear Programming technique. A route cannot be simply chosen just as it will most minimize time or it will cover the least possible distance. Examples with requirements to consider the degree of rejection or hesitation (or both) are overflowing in our materialistic world. Here comes the need to consider TSP under intuitionistic fuzzy environment. The degree of rejection as well as the degree of hesitancy must be studied to find the solution in a truly optimum sense! Proposed technique is an extension as well as collaboration of ideas of fuzzy traveling salesperson problem and intuitionistic fuzzy (IF) optimization technique.

\section{Indexing terms/Keywords}

Travelling salesman problem; intuitionistic fuzzy; degree of hesitation; fuzzy optimization; intuitionistic fuzzy decision making.

\section{Academic Discipline And Sub-Disciplines}

Mathematics; Optimization and Operation Research; Computer Science;

\section{SUBJECT CLASSIFICATION}

AMS Classification: Fuzzy programming 90C70; Operations research and management science 90Bxx; Decision Theory and Fuzziness 62C86;

\section{TYPE (METHOD/APPROACH)}

Intuitionistic Fuzzy modeling in solving well known travelling salesperson problem.

\section{Council for Innovative Research}

Peer Review Research Publishing System

Journal: INTERNATIONAL JOURNAL OF COMPUTERS \& TECHNOLOGY

Vol 11, No. 9

editor@cirworld.com

www.cirworld.com. member.cirworld.com 


\section{INTRODUCTION}

"Mathematical discovery is like feeling your way in a dark room: putting your hand here and finding a chair, putting your hand there and finding a table... At some point, your hand hits the light switch, and suddenly everything makes sense. For the traveling salesman problem, even after so many papers that have pushed the envelope in so many directions, I don't think that we have hit that switch yet." Amin Saberi (Stanford University).

The term "travelling salesman problem" (TSP) [9] is a non-deterministic polynomial-time hard problem in combinatorial optimization studied in graph theory, operations research and theoretical computer science. Given a list of cities and their pair wise distances, the task is to find the shortest possible route that visits each city exactly once and returns to the origin city [10]. The origins of the travelling salesman problem are unclear. The travelling salesman problem was defined in the 1800 s by the Irish mathematician W. R. Hamilton and by the British mathematician Thomas Kirkman. Great progress was made in the late 1970s and 1980, when Grötschel, Padberg, Rinaldi and others managed to exactly solve instances with up to 2392 cities, using cutting planes and branch-and-bound. In 2005, Cook and others computed an optimal tour through a 33,810-city instance given by a microchip layout problem, one of the largest solved instances. For many other instances with millions of cities, solutions can be found that are guaranteed to be within $1 \%$ of an optimal tour [10].

Suppose a salesman wants to visit $\mathrm{n}$ cities. The distances (or cost or time or any other parameter) of journey between any two cities is known to him. He starts from a particular city and passes through each city exactly once and returns to his original city. Hence the salesman may choose from $(n-1)$ ! different possible route. The problem is to find the optimum. It is also known as routing problem. The optimum solution is independent of the selection of the starting city $[7,8]$.

By decision-making in a fuzzy environment is meant a decision process in which the goals and/or the constraints, but not necessarily the system under control, are fuzzy in nature [4]. A list of publications that uses fuzzy set to solve TSP is given in table 1. (The list is not of exhaustive nature) [7].

Table1. Some of the recent solution strategies in the literature for TSP

\begin{tabular}{|c|c|c|}
\hline Author(s) & Year of Publication(s) & Strategy \\
\hline Espied Fereidouni & 2011 & Fuzzy multi- objective linear programming \\
\hline Chaudhuri et al. & 2011 & Fuzzy multi- objective linear programming \\
\hline Rehmat et al. & 2007 & \begin{tabular}{c} 
Fuzzy multi- objective linear programming \\
\hline Paquete et al.
\end{tabular} \\
\hline Angel et al. & 2004 & $\begin{array}{c}\text { Pareto local search method which } \\
\text { extended local search algorithm }\end{array}$ \\
\hline Yan et al. & 2004 & Dynamic search algorithm by local search \\
\hline Jaszkiewicz & 2003 & Evolutionary algorithm \\
\hline
\end{tabular}

For Multi-Objective Symmetric TSP, in fuzzy environment, it is converted into a Linear Program using Fuzzy MultiObjective Linear Programming (FMOLP) technique. The route selection of problem is done by exploiting aspiration level parameters.

Now, it may be noted that in route selection, selection of some particular route may lead to some real practical difficulties. We may not always select a route just because it will most minimize time or it will cover the least possible distance. A recent survey report of 300 businesses across the U.S. and Canada states "...employees really have been spending more time at the office since the recession hit back in 2008... Almost two-thirds of workers spend more time at work than three years ago...", lifeinc.today.com found. "Employees generally don't mind doing more with less especially when economic conditions are tough," said Ryan Johnson, Vice President of Research for WorldatWork. Though companies expressed concern about the extra hours, they aren't offering financial rewards for employees boost in work. Hence, sometimes, for companies as well as for employees, cost cutting is more important than anything else, even if selection of that route may consume more time! So, tendency of rejecting an allotment (i.e. degree of rejection of cost over and above some level) needs to be checked. Again, to select the travel-route of our president, security along a road is an important criterion. Hence, traveling by road along a stretch in a terrorist zone, to make the cost minimum most, will be a decision with hesitation to some extent (i.e. in that case, the budgetary allocation will be made flexible in nature). Similarly, flying along a route, just because it cuts time and distance, but where it is raining heavily, may not get approval from appropriate authority (somewhat rejected). Examples with requirements to consider the degree of rejection or hesitation (or both) are overflowing in our materialistic world. Here comes the need to consider TSP under intuitionistic fuzzy environment [2]. The degree of rejection as well as the degree of hesitancy must be studied to find the solution in a truly optimum sense!

Our technique is an extension as well as collaboration and mixture of ideas from fuzzy traveling salesperson problem (FTSP) [6] and intuitionistic fuzzy (IF) optimization technique [1]. The IF technique to solve intuitionistic fuzzy optimization problem was first proposed by Plamen P. Angelov in 1997 [1] and it is widely considered as a successful tool by researchers all over the world. It is well known that the advantages of the intuitionistic fuzzy optimization problems are 
twofold: firstly, they give the richest apparatus for formulation of optimization problems and on the other hand, the solution of intuitionistic fuzzy optimization problems may satisfy the objective(s) with bigger degree of satisfaction than the analogous fuzzy optimization problem and the crisp one. Angelov's approach is an application of the intuitionistic fuzzy (IF) set concept to optimization problems. In his approach, the degree of acceptance is maximized while the degree of rejection is minimized. In our approach, not only the degree of acceptance is maximized and the degree of rejection is minimized but also the degree of hesitation is minimized [1].

The rest of this paper is organized as follows. In the next section, some basic definitions are given. Next, solution method of fuzzy multi objective travelling salesman problem is discussed. In next section, proposed intuitionistic fuzzy optimization technique is given. Next, a numerical example of proposed solution procedure on intuitionistic fuzzy travelling salesperson problem (IFTSP) is discussed and finally, a comparison is given among different circumstances and conclusion is made.

\section{DEFINITIONS}

A fuzzy subset $\tilde{A}$ of $X$ is defined by its membership function $\mu \tilde{A}: X \rightarrow[0,1]$, that assigns to every $x \in X$, a real number $\mu_{\bar{A}}(x)$ in the closed unit interval $[0,1]$, where the value of $\mu \tilde{A}(x)$ at $x$ represents the grade of membership of $x$ in $\tilde{A}$.

On the other hand, an intuitionistic fuzzy set $A$ in $X$ is defined by $A=\left\{<x ; \mu_{A}(x), v_{A}(x)>\mid x \in E\right\}$, where $\mu_{A}: E \rightarrow[0,1]$ and $\mathrm{v}_{\mathrm{A}}: \mathrm{E} \rightarrow[0,1]$ with the condition $0 \leq\left(\mu_{\mathrm{A}}(\mathrm{x})+\mathrm{v}_{\mathrm{A}}(\mathrm{x})\right) \leq 1$, where $\mu_{\mathrm{A}}(\mathrm{x})$ and $\mathrm{v}_{\mathrm{A}}(\mathrm{x})$ denote the degree of membership and non membership respectively. It is clear that for every fuzzy set $\tilde{A}$, there exist an intuitionistic fuzzy set $A=\left\{<x ; \mu_{A}(x), 1-\mu_{A}\right.$ $(x)>\mid x \in E\}$. Also, for each intuitionistic fuzzy set in $X$, we set $\pi_{A}(x)=1-\mu_{A}(x)-v_{A}(x)$ and it is called the degree of hesitancy or hesitation index or intuitionistic index of $x$ in $A$. It is clear that $0 \leq \pi_{A}(x) \leq 1$ for each $x$ in universal set.

An intuitionistic fuzzy subset of the real line is called an Intuitionistic fuzzy number $\tilde{A}^{i}$ if it is (a) normal i.e. there is any $x_{0} \in$ $\mathrm{R}$ such that $\mu_{\tilde{A}^{i}}\left(\mathrm{x}_{0}\right)=1$ (and so $\mathrm{v}_{\mathrm{A}}\left(\mathrm{x}_{0}\right)=0$ ), (b) convex set for the membership function i.e. $\mu_{\tilde{A}^{i}}\left(\lambda x_{1}+(1-\lambda) x_{2}\right) \leq \min \left(\mu_{\tilde{A}^{i}}\left(x_{1}\right), \mu_{\tilde{A}^{i}}\left(x_{2}\right)\right), \forall \mathrm{x}_{1}, \mathrm{x}_{2} \in \mathrm{R}, \lambda \in[0,1]$ and (c) concave set for the non-membership function i.e. $v_{\tilde{A}^{i}}(x)$ i.e. $\quad v_{\tilde{A}^{i}}\left(\lambda x_{1}+(1-\lambda) x_{2}\right) \geq \max \left(v_{\tilde{A}^{i}}\left(x_{1}\right), v_{\tilde{A}^{i}}\left(x_{2}\right)\right), \forall \mathrm{x}_{1}, \mathrm{x}_{2} \in \mathrm{R}, \lambda \in[0,1]$.

\section{FUZZY MULTI OBJECTIVE OPTIMIZATION TECHNIQUE TO SOLVE TSP}

Deterministic optimization problems are well studied, but they are very limited and in many cases they do not represent exactly the real problem. Usually, it is difficult in describing the constraints of an optimization problem by strict crisp relations (equalities and/or non-equalities). Practically, a small violation of a given constraint is admissible and it can lead to a more efficient solution of the real problem. In over the last three decades, optimization problems have been investigated in the sense of fuzzy set theory. Fuzzy optimization formulations are more flexible and allow finding solutions that are more adequate to the real problem in comparison with crisp problems.

Let us consider any symmetric (objective may be used as constraint and vice versa) crisp multi objective optimization problem of the form: $\quad$ Minimize $\quad f_{i}(x), \quad i=1 \ldots p$,

subject to the constraints

$$
g_{j}(x) \leq 0, \quad j=1 \ldots q .
$$

Here $x$ denotes the unknown variables, $f_{i}(x)$ are the objective functions, $g_{j}(x)$ are the constraints (non-equalities), $p$ denotes the number of objectives and q denotes the number of constraints. The solution of this crisp optimization problem satisfies all constraints exactly. In the analogous fuzzy optimization problem, the degree of satisfaction of objective(s) as well as of constraint(s) is maximized. The problem is of the form:

$$
\text { Minimize } \quad \mathrm{f}_{\mathrm{i}}(\mathrm{x}), \quad \mathrm{i}=1 \ldots \mathrm{p} \text {, }
$$

subject to the constraints

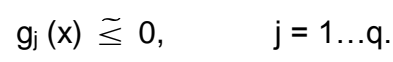

Here Minimize denotes the fuzzy minimization and $\tilde{\leq}$ denotes the fuzzy inequality [4]

Next, it is transformed via Zimmerman method and Bellman-Zadeh's approach in decision making under fuzzy environment, hat gives the following optimization problem: to maximize the degree of membership (acceptance) of the objective(s) as well as of the constraints to the respective fuzzy sets:

$$
\begin{aligned}
& \operatorname{Max}_{i}(x), x \in R^{n}, i=1 \ldots(p+q), \\
& \text { subject to the constraints } 0 \leq \mu \mathrm{i}(\mathrm{x}) \leq 1,
\end{aligned}
$$

where $\mu \mathrm{i}(\mathrm{x})$ denotes degree of acceptance of $\mathrm{x}$ in $R^{n}[4]$. 
The most frequently considered objective of TSP is to determine an optimal order for traveling all cities so that total cost, distance, time is minimized. Consider the situation when decision maker has to determine optimal solution of TSP with minimized cost, time and overall distance. The individual objective functions can be formed for all objectives of decision maker. Let $x_{i j}$ be the link from city $\mathrm{i}$ to $\mathrm{j}$ and

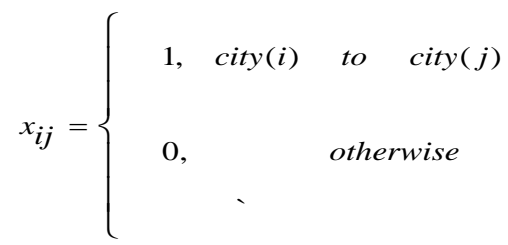

Let $c_{i j}$ be the cost of travelling from city $\mathrm{i}$ to $\mathrm{j}$; overall cost of particular route is sum of costs on links comprising the route. Since, decision maker has to minimize overall traveling cost the goal can be set for total estimated cost of entire route for TSP denoted by $z_{1}^{0}$. But there can be situations when estimated cost doesn't meet and so decision maker can set acceptance level of tolerance for estimated cost. Denoting tolerance against this goal as $t_{1}$, objective function for minimization of cost is given as follows:

$$
z_{1}: \min \sum_{i=1}^{n} \sum_{j=1}^{n} c_{i j} x_{i j} \leq z_{1}^{0}
$$

Let $\mathrm{d}_{\mathrm{ij}}$ be the distance from city $\mathrm{i}$ to $\mathrm{j}$ and $z_{2}^{0}$ be the corresponding aspiration level for objective function for minimization of distance and $t_{2}$ be the acceptance level of tolerance, then objective function takes following form:

$$
z_{2}: \min \sum_{i=1}^{n} \sum_{j=1}^{n} d_{i j} x_{i j} \leq z_{2}^{0}
$$

Let $t_{i j}$ be the time spent in travelling from city $\mathrm{i}$ to $\mathrm{j}$ and $z_{3}^{0}$ be the corresponding aspiration level for objective function for minimization of total time and $t_{3}$ be acceptance level of tolerance. The objective function is written as follows:

$$
z_{3}: \min \sum_{i=1}^{n} \sum_{j=1}^{n} t_{i j} x_{i j} \leq z_{3}^{0}
$$

It is noted that dependency of objective functions on each other is one important aspect of TSP. They are mostly dependent, but determining exact form of dependency is complex process. The fuzzy TSP framework works in all cases, if there is some feasible solution, as suggested by Chaudhuri, Arindam et al. In their method, these multiple objective functions are represented in vector form comprising multiple objectives with specified goals and tolerances. The membership functions are set for these individual objective functions to check their level of acceptability. The restriction that every city be visited from exactly one location and vice versa generates the constraints:

$$
\sum_{i=1}^{n} x_{i j}=1, \forall j \text { and } \sum_{j=1}^{n} x_{i j}=1, \forall i \text {. }
$$

Also, since a route is selected at most once, the constraints are $x_{i j}+x_{j i} \leq 1, \forall i, j$, and the non negativity constraints: $\mathrm{x}_{\mathrm{ij}}$ is non-negative. These constraints collectively are expressed in vector form and fuzzy membership functions are defined for all objective functions. Finally, linear model is formulated using fuzzy multiobjective linear programming (FMOLP) model using TSP objective functions, their corresponding membership functions and constraints $[6,7,8]$.

\section{PROPOSED INTUITIONISTIC FUZZY OPTIMIZATION (IFO) TECHNIQUE}

Fuzzy set theory has been widely developed and various modifications and generalizations have appeared. One of them is the concept of intuitionistic fuzzy (IF) set [2,3]. It considers not only the degree of membership to a given set, but also the degree of rejection such that the sum of both these values is less than or equal to one. Complement of this sum to ' 1 ' is known as intuitionistic fuzzy index (degree of hesitation). Applying this concept Angelov, in 1997, has reformulated the fuzzy optimization problem.

Angelov has well identified that, in general, an optimization problem includes objective(s) and constraint(s). In fuzzy optimization problems, the objective(s) and/or constraint(s) or parameter(s) and relation(s) are represented by fuzzy sets. The solution of crisp optimization problem must satisfy all constraints exactly. And in the analogous fuzzy optimization problem, the degree of satisfaction of objective(s) and of constraint(s) is maximized. It is transformed via Bellman-Zadeh's approach. In the case when the degree of rejection (non-membership) is defined simultaneously with the degree of acceptance (membership) and when both these degrees are not complementary to each other, IF sets can be used as a more general and complete tool for describing this uncertainty. 
Yet, in Angelov's paper, the degree of hesitation is not used at all. It must be noted that Szmidt and Kacprzyk have already mentioned, in 2000, that taking into account the third parameter (degree of hesitation) when calculating the Euclidean distance for intuitionistic fuzzy sets does have an influence on the final result [5]. It should be so because a two dimensional geometric interpretation is an orthogonal projection of a real situation as shown suitably in their historic paper. Also the output is consistent as desired. Moreover, in a decision making process it is desired that there will be minimal hesitation in the final decision. The final outcome may be either acceptable or not acceptable but if it has a bigger degree of hesitancy; it will be difficult for the decision maker to come to a well acceptable conclusion. Hence, we plan to minimize the degree of hesitation as well. Having in mind the above mentioned facts, in order to be more concordant with the mathematical notion, we have used this third parameter, degree of hesitation, in the proposed method of our study.

Next, if in an optimization problem $G$ denotes goal and $C$ denotes constraints, we have the decision $D$ defined by

$$
D=G \cap C=\left\{<x, \mu_{G}(x) \cap \mu_{C}(x), v_{G}(x) \cup v_{C}(x)>: x \in R^{n}\right\} .
$$

This operator can be easily generalized and applied to the IFO problem [1]:

$$
\begin{aligned}
& \left.D=\left\{<x, \mu_{D}(x), v_{D}(x), \pi_{D}(x)\right)>: x \in R^{n} \text { and } \pi_{D}(x)=1-\left(\mu_{D}(x)+v_{D}(x)\right)\right\}, \mu_{D}=\bigcap_{i=1}^{p+q} \mu_{i}, v_{D}=\bigcup_{i=1}^{p+q} v_{i}, \text { with } \\
& \mu_{D}=\min _{i=1}^{p+q} \mu_{i}, x \in R^{n}, \mu_{D} \leq \mu_{i} \text { and } v_{D}=\max _{i=1}^{p+q} v_{i}, x \in R^{n}, v_{D} \geq v_{i} .
\end{aligned}
$$

So, to maximize the degree of acceptance of IF objectives and constraints and to minimize the degree of rejection of IF objectives and constraints and to minimize the degree of hesitation of IF objectives and constraints, the following constraints need to be solved:

$$
\begin{aligned}
& \alpha \leq \mu_{i}(x), \quad i=1,2, \ldots,(p+q), \\
& \beta \geq v_{i}(x), \quad i=1,2, \ldots,(p+q), \\
& \gamma \geq 1-\mu_{i}(x)-v_{i}(x), i=1,2, \ldots,(p+q), \\
& \alpha \geq \beta, \quad \beta \geq \gamma, \quad \alpha+\beta+\gamma=1, \\
& \alpha, \beta, \gamma \geq 0, \quad \text { other crisp constraints and } \\
& \text { non negativity retrstrictions of variables, }
\end{aligned}
$$

Here, $\alpha(0 \leq \alpha \leq 1)$ denotes the minimal acceptable limit of degree of objective(s) and constraints and $\beta(0 \leq \beta \leq 1)$ denotes the maximal degree of rejection of objective(s) and constraints and $\gamma \quad(0 \leq \gamma \leq 1)$ denotes the maximal allowable degree of hesitation of objective(s) and constraints (that are IF in nature)

Thus, the IFO problem is transformed to the following crisp (non-fuzzy) optimization problem which can be easily solved numerically or by using any standard software:

$$
\begin{aligned}
& \text { Maximize } \quad \alpha-\beta-\gamma \\
& \text { subject to the constraints } \alpha \leq \mu_{i}(x), i=1,2, \ldots,(p+q), \\
& \beta \geq v_{i}(x), \quad i=1,2, \ldots,(p+q), \\
& \gamma \geq 1-\mu_{i}(x)-v_{i}(x), \quad i=1,2, \ldots,(p+q), \\
& \alpha \geq \beta, \quad \beta \geq \gamma, \quad \alpha+\beta+\gamma=1, \\
& \alpha, \beta, \gamma \geq 0
\end{aligned}
$$

and other crisp constraints and non-negativity restrictions

\section{NUMERICAL SOLUTION OF INTUITIONISTIC FUZZY TRAVELLING SALESPERSON PROBLEM (IFTSP)}

Traveling salesman problem is analyzed for symmetric TSP. Let the salesman starts from his home city 0 ; has to visit the three cities (city 1 , city 2 , city 3 ) exactly once and he is required to come back to his home city 0 by adopting a route with minimum cost, time and distance covered (Chaudhuri and De, 2011). The cities are listed along with their cost, time and distance in a matrix below, where triple (c, $\mathrm{d}, \mathrm{t}$ ) represents: cost in dollars (in hundreds), distance in kilo meters, and time in hours respectively for the corresponding couple of cites [6, 7]. 
Table 2. The matrix for time, cost and distance for each pair of cities

\begin{tabular}{|c|c|c|c|c|}
\hline & $\begin{array}{c}\text { (Cost,Distance,Time) } \\
(\mathbf{c}, \mathbf{d}, \mathbf{t})\end{array}$ & $\begin{array}{c}\text { (Cost,Distance,Time) } \\
(\mathbf{c}, \mathbf{d}, \mathbf{t})\end{array}$ & $\begin{array}{c}\text { (Cost,Distance,Time) } \\
\mathbf{( c ,}, \mathbf{d}, \mathbf{t})\end{array}$ & $\begin{array}{c}\text { (Cost,Distance, } \\
\text { Time) (c, } \mathbf{d}, \mathbf{t})\end{array}$ \\
\cline { 2 - 5 } & $\mathbf{0}$ & $\mathbf{1}$ & $\mathbf{2}$ & $\mathbf{3}$ \\
\hline $\mathbf{0}$ & $(0,0,0)$ & $(20,5,4)$ & $(15,5,5)$ & $(11,3,2)$ \\
\hline $\mathbf{1}$ & $(20,5,4)$ & $(0,0,0)$ & $(30,5,3)$ & $(20,10,2)$ \\
\hline $\mathbf{2}$ & $(15,5,5)$ & $(30,5,3)$ & $(20,0,0)$ & $(0,0,0)$ \\
\hline $\mathbf{3}$ & $(11,3,2)$ & $(10,3,3)$ & & \\
\hline
\end{tabular}

In this case, we first have to formulate the three objective function $z_{1}, z_{2}, z_{3}$ for cost, distance and time respectively. From the table, we have the objective functions as follows:

$z_{1}=20^{*} x 01+15^{\star} x 02+11^{*} x 03+20^{*} x 10+30^{*} x 12+10^{*} x 13+15^{\star} x 20+30^{*} x 21+20^{*} x 23+11^{*} x 30+10^{\star} x 31+20^{*} x 32, z_{2}$ $=5^{*} x 01+5^{\star} x 02+3^{*} x 03+5^{*} x 10+5^{\star} x 12+3^{*} x 13+5^{*} x 20+5^{*} x 21+10^{*} x 23+3^{*} x 30+3^{*} x 31+10^{*} x 32, z_{3}=4^{*} x 01+5^{*} x 02$ $+2^{*} x 03+4^{*} x 10+3^{*} x 12+3^{*} x 13+5^{*} x 20+3^{*} x 21+2^{*} x 23+2^{*} x 30+3^{*} x 31+2^{*} x 32$.

The aspiration levels are set as 65,16 and 11 by solving each objective function separately subject to the given constraints in the TSP and their corresponding tolerances level of acceptance i.e. the degrees of acceptance are decided as $5,2,4$ respectively (by the decision maker). We also decide $9,4,5$ to be the respective tolerance level for rejection i.e. degree of rejection of the objective functions. Thus the respective membership functions and non-membership functions of the objective functions $z_{1}, z_{2}, z_{3}$ may be written as follows:

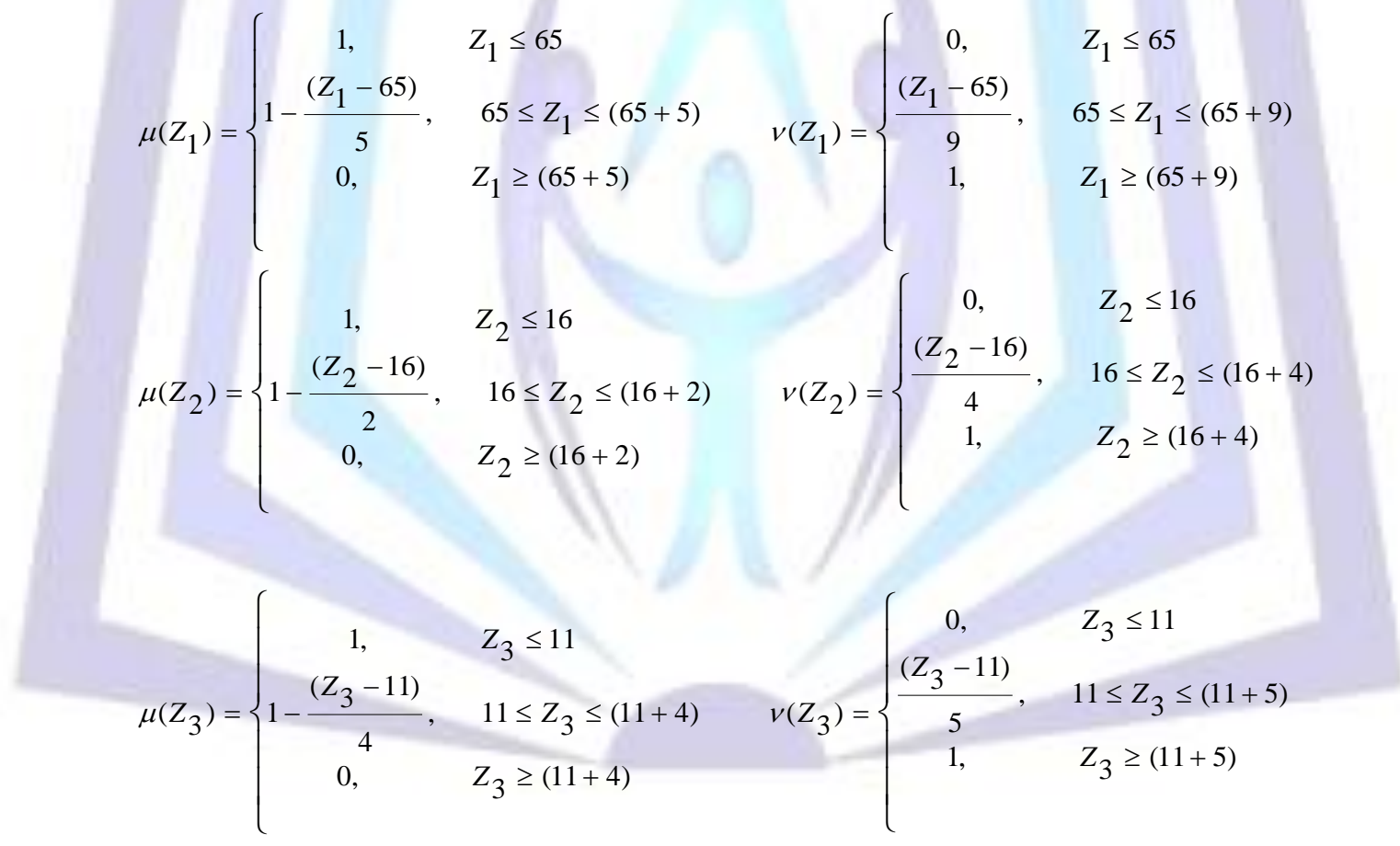

Hence, in order to maximize the degree of acceptance, to minimize the degree of rejection and to minimize the degree of hesitation, the IFTSP takes the following form:

$$
\operatorname{Max} \alpha-\beta-\gamma,
$$

subject to the constraints

$$
\begin{aligned}
& \mu\left(z_{1}\right) \geq \alpha, \mu\left(z_{2}\right) \geq \alpha, \mu\left(z_{3}\right) \geq \alpha, \\
& \nu\left(z_{1}\right) \leq \beta, \nu\left(z_{2}\right) \leq \beta, \nu\left(z_{2}\right) \leq \beta, \\
& 1-\left(\mu\left(z_{1}\right)+\nu\left(z_{1}\right)\right) \leq \gamma, \\
& 1-\left(\mu\left(z_{2}\right)+\nu\left(z_{2}\right)\right) \leq \gamma, \\
& 1-\left(\mu\left(z_{3}\right)+\nu\left(z_{3}\right)\right) \leq \gamma, \\
& \alpha \geq \lambda, \beta \geq \gamma, \alpha \geq \beta, \alpha+\beta+\gamma=1,
\end{aligned}
$$




$$
\begin{aligned}
& x_{01}+x_{02}+x_{03}=1, x_{11}+x_{12}+x_{13}=1, \\
& x_{21}+x_{21}+x_{23}=1, x_{30}+x_{31}+x_{32}=1, \\
& x_{10}+x_{20}+x_{30}=1, x_{01}+x_{21}+x_{31}=1, \\
& x_{02}+x_{12}+x_{32}=1, x_{03}+x_{13}+x_{23}=1, \\
& x_{01}+x_{10} \leq 1, x_{02}+x_{20} \leq 1, x_{03}+x_{30} \leq 1, \\
& x_{12}+x_{21} \leq 1, x_{13}+x_{31} \leq 1, x_{23}+x_{32} \leq 1, \\
& \alpha, \beta, \gamma \geq 0, x_{i j} \geq 0, \forall i, j=0,1,2,3
\end{aligned}
$$

It is noted that the membership, non membership functions are linear. First, we omit the intuitionistic fuzzy sense and consider only the fuzzy sense, i.e. if only the membership function of degree of acceptance is considered by removing other two, we get fuzzy travelling salesman problem, which upon conversion to crisp sense (Bellman-Zadeh method and Zimmerman method) gives the following linear programming problem:

Maximize $\alpha$,

subject to the constraints

$$
\begin{aligned}
& 1-\frac{\left(Z_{1}-65\right)}{5} \geq \alpha, 1-\frac{\left(Z_{2}-16\right)}{2} \geq \alpha, 1-\frac{\left(Z_{3}-11\right)}{4} \geq \alpha, \\
& x_{\mathrm{O} 1}+x_{\mathrm{O} 2}+x_{\mathrm{O} 3}=1, x_{11}+x_{12}+x_{13}=1, \\
& x_{21}+x_{21}+x_{23}=1, x_{30}+x_{31}+x_{32}=1, \\
& x_{10}+x_{20}+x_{30}=1, x_{\mathrm{O} 1}+x_{21}+x_{31}=1, \\
& x_{\mathrm{O} 2}+x_{12}+x_{32}=1, x_{\mathrm{O} 3}+x_{13}+x_{23}=1, \\
& x_{\mathrm{O} 1}+x_{10} \leq 1, x_{\mathrm{O} 2}+x_{20} \leq 1, x_{\mathrm{O} 3}+x_{30} \leq 1, \\
& x_{12}+x_{21} \leq 1, x_{13}+x_{31} \leq 1, x_{23}+x_{32} \leq 1, \\
& \alpha \geq 0, x_{i j} \geq 0, \forall i, j=0,1,2,3
\end{aligned}
$$

Solution using Lingo [win32 v. 13.0.2.16(11 Apr. 2012) (API: 7.0.1.448)] in a computer having AMD dual-Core Processor with 2 GB DDR3 RAM gives the result: $\alpha=0.54$ and if $z=z 1^{*}+z 2^{*}+z 3^{*}$, it gives $z=67.28+16.60+12.83=96.71$.

Again, if we solve the same problem in Angelov's method of intuitionistic fuzzy optimization method, i.e. the degree of acceptance is maximized, the degree of rejection is minimized, ignoring the degree of hesitation completely, the problem becomes:

\section{Maximize $\quad \alpha-\beta$,}

subject to the constraints

$$
\begin{aligned}
& 1-\frac{\left(Z_{1}-65\right)}{5} \geq \alpha, 1-\frac{\left(Z_{2}-16\right)}{2} \geq \alpha, 1-\frac{\left(Z_{3}-11\right)}{4} \geq \alpha, \\
& \frac{\left(Z_{1}-65\right)}{9} \leq \beta, \frac{\left(Z_{2}-16\right)}{4} \leq \beta, \frac{\left(Z_{3}-11\right)}{5} \leq \beta, \\
& \alpha \geq \beta, \alpha+\beta \leq 1, \\
& x_{\mathrm{O} 1}+x_{\mathrm{O} 2}+x_{\mathrm{O} 3}=1, x_{11}+x_{12}+x_{13}=1, \\
& x_{21}+x_{21}+x_{23}=1, x_{30}+x_{31}+x_{32}=1, \\
& x_{10}+x_{20}+x_{30}=1, x_{\mathrm{O} 1}+x_{21}+x_{31}=1, \\
& x_{\mathrm{O} 2}+x_{12}+x_{32}=1, x_{\mathrm{O} 3}+x_{13}+x_{23}=1, \\
& x_{\mathrm{O} 1}+x_{10} \leq 1, x_{\mathrm{O} 2}+x_{20} \leq 1, x_{\mathrm{O} 3}+x_{30} \leq 1, \\
& x_{12}+x_{21} \leq 1, x_{13}+x_{31} \leq 1, x_{23}+x_{32} \leq 1, \\
& \alpha, \beta \geq 0, x_{i j} \geq 0, \forall i, j=0,1,2,3
\end{aligned}
$$

Solution, in same procedure and under same technical environment, gives the result: $\alpha=0.54, \beta=0.36$ and if $z^{\prime}=z 1^{*}+$ $z 2^{*}+z 3^{*}$, it gives $z^{\prime}=67.28+16.60+12.83=96.71$, which is same as fuzzy result. Now, finally, we consider the degree of hesitation as well; giving to the membership and non-membership and intuitionistic index functions, the problem becomes 


$$
\begin{aligned}
& \text { Maximize } \quad \alpha-\beta-\gamma, \\
& \text { subject to the constraints } \\
& 1-\frac{\left(Z_{1}-65\right)}{5} \geq \alpha, 1-\frac{\left(Z_{2}-16\right)}{2} \geq \alpha, 1-\frac{\left(Z_{3}-11\right)}{4} \geq \alpha, \\
& \frac{\left(Z_{1}-65\right)}{9} \leq \beta, \frac{\left(Z_{2}-16\right)}{4} \leq \beta, \frac{\left(Z_{3}-11\right)}{5} \leq \beta, \\
& 1-\left(\left(1-\frac{\left(Z_{1}-65\right)}{5}\right)+\left(\frac{\left(Z_{1}-65\right)}{9}\right)\right) \leq \gamma, \\
& 1-\left(\left(1-\frac{\left(Z_{2}-16\right)}{2}\right)+\left(\frac{\left(Z_{2}-16\right)}{4}\right)\right) \leq \gamma, \\
& 1-\left(\left(1-\frac{\left(Z_{3}-11\right)}{4}\right)+\left(\frac{\left(Z_{3}-11\right)}{5}\right)\right) \leq \gamma, \\
& \alpha \geq \lambda, \beta \geq \gamma, \alpha \geq \beta, \alpha+\beta+\gamma=1, \\
& x_{\mathrm{O} 1}+x_{\mathrm{O} 2}+x_{\mathrm{O} 3}=1, x_{11}+x_{12}+x_{13}=1, \\
& x_{21}+x_{21}+x_{23}=1, x_{30}+x_{31}+x_{32}=1, \\
& x_{10}+x_{20}+x_{30}=1, x_{\mathrm{O} 1}+x_{21}+x_{31}=1, \\
& x_{02}+x_{12}+x_{32}=1, x_{\mathrm{O} 3}+x_{13}+x_{23}=1, \\
& x_{\mathrm{O} 1}+x_{10} \leq 1, x_{\mathrm{O} 2}+x_{20} \leq 1, x_{\mathrm{O} 3}+x_{30} \leq 1, \\
& x_{12}+x_{21} \leq 1, x_{13}+x_{31} \leq 1, x_{23}+x_{32} \leq 1, \\
& \alpha, \beta, \gamma \geq 0, x_{i j} \geq 0, \forall i, j=0,1,2,3
\end{aligned}
$$

\begin{tabular}{|c|c|c|c|c|c|}
\hline TSP Method & $\begin{array}{l}\text { Tolerance } \\
\text { level for } \\
\text { rejection } \\
t_{1}{ }^{\prime}, t_{2}{ }^{\prime}, t_{3}{ }^{\prime}\end{array}$ & $\mathbf{R}$ & $\begin{array}{l}\text { Output } \\
\alpha, \beta, Y\end{array}$ & $\begin{array}{c}\text { Optimum } \\
\text { route of } \\
\text { travel }\end{array}$ & Total Sum $(z)=z_{1}+z_{2}+z_{3}$ \\
\hline $\begin{array}{l}\text { Angelov's } \\
\text { Method }\end{array}$ & $9,4,5$ & $E$ & $0.54,0.36$ & $\mathrm{x}_{02}, \mathrm{x}_{21}, \mathrm{x}_{13}$ & $67.28+16.60+12.83=96.71$ \\
\hline $\begin{array}{l}\text { Proposed } \\
\text { Method }\end{array}$ & $9,4,5$ & $\mathbf{U}$ & $0.50,0.400 .10$ & $x_{03}, x_{31}, x_{12}$ & $66.11+16.05+12.99=95.15$ \\
\hline \multicolumn{2}{|c|}{ Results while ... } & & \multicolumn{3}{|c|}{... taking another set of tolerances } \\
\hline $\begin{array}{l}\text { Angelov's } \\
\text { Method: }\end{array}$ & $7,6,8$ & $\mathbf{L}$ & $0.52,0.24$, & $\mathrm{x}_{02}, \mathrm{x}_{21}, \mathrm{x}_{13}$ & $66.67+16.31+12.91=95.89$ \\
\hline $\begin{array}{l}\text { Proposed } \\
\text { Method: }\end{array}$ & $7,6,8$ & $\mathbf{T}$ & $0.52,0.24,0.24$ & $x_{02}, x_{21}, x_{13}$ & $66.67+16.31+12.91=95.89$ \\
\hline
\end{tabular}

The intuitionistic fuzzy linear travelling salesperson problem, thus converted into a crisp linear programming, is now solved using same software in same machine. The result gives $\alpha=0.50, \beta=0.40$ and $y=0.10$ and if $z=z 1^{*}+z 2^{*}+z 3^{*}$, it gives $z^{\prime \prime}=66.11+16.05+12.99=95.15$, which is better than fuzzy result as well as Angelov's intuitionistic fuzzy result (both are same in our case).

It is noted that this property of our IFO problem is analogous to the property of fuzzy optimization problems when in some cases (it depends on the particular problem) the solution can satisfy the objective function "better", but the price for this is the "worse" satisfaction of some constraints.

We now treat this problem in different circumstances and compare the results in following table.

Table 3. Comparison among examples under different circumstances 


\section{CONCLUSIONS AND FUTURE WORK}

Are results interesting to note? The cost is higher in Angelov's method in comparison with proposed method. But, the degree of acceptance is higher in Angelov's method. And, the optimum routes are different among these two processes. If small (here, only 04 out of 100) difference in degree of acceptance is ignored, and we like to find the degree of hesitation as well, prioritizing minimization of cost, proposed method scores better. But, if degree of acceptance is top most priority, Angelov's method makes higher score. As already discussed, in the period of global economic downfall, cost cutting is very important for every organization. Also, employees are willing to work few extra minutes to tide over this recession period. Hence, from company's perspective, minimization cost in exchange of few working hours is more suitable.

It is also noted that if we change the level of tolerances to 7, 6, 8 respectively, the results in both methods coincide. Thus, using Angelov's realization, this property of the IFO problem is analogous to the property of fuzzy optimization problems when in some cases (it depends on the membership function) the solution can satisfy the objective function "better", but the price for this is the "worse" satisfaction of some constraints, whereas same results are obtained in some cases also. The tolerances are introduced by decision maker to accommodate this vagueness. By adjusting these tolerances, a range of solutions with different aspiration levels are obtained from which decision maker chooses one that best meets his satisfactory level within given tolerances. There is a definite potential for further work on development of methods to solve TSP problems with vague description of resources. As Arindam et. all well said, for efficient results, some heuristics are required such as relative dependencies among objective functions.

\section{ACKNOWLEDGMENTS}

The first author will like to thank Dr. Murari Mitra and Dr. Basudeb Mukherjee, Department of Mathematics, BESU, for their valuable comments, advises and suggestions all through last seven years.

\section{REFERENCES}

[1] Angelov, P. Plamen. 1997 Optimization in an intuitionistic fuzzy environment. Fuzzy Sets and Systems. Vol. 86, 299306.

[2] Atanassov, K. 1986 Intuitionistic fuzzy sets. Fuzzy Sets and Systems. Vol. 20, 87-96.

[3] Atanassov, K. 1995 Ideas for intuitionistic fuzzy sets equations, inequalities and optimization. Notes on Intuitionistic Fuzzy Sets. Vol. 1, No. 1, 17-24.

[4] Bellman, R. E. and L. A. Zadeh. 1970 Decision making in a fuzzy environment. Management Science. Vol. 17, B141B164.

[5] Szmidt, E. and Kacprzyk, J. 1997 Distances between intuitionistic fuzzy sets. Fuzzy Sets and Systems. Vol. 114, 505-518.

[6] Chaudhuri, Arindam and Kajal, De. 2011 Fuzzy multi-objective linear programming for travelling salesman problem. African Journal of Mathematics and Computer Science Research. Vol. 4(2) (Feb. 2011), 64-70.

[7] Fereidouni, Sepideh. 2011 Solving travelling salesman problem by using a multi-objective linear programming. African Journal of Mathematics and Computer Science Research. Vol. 4(11) (Oct. 2011), 339-349.

[8] Bektas, Tolga. 2006 The multiple travelling salesman problems: an overview of formulations and solution procedures. Omega. Vol. 34, 209-219.

[9] Hoffman, P. 1998 The Man Who Loved Only Numbers: The Story of Paul Erdős and the Search for Mathematical Truth. New York: Hyperion, 168-169.

[10] Wikipedia Contributors. 2013 Intuitionism. Wikipedia, The Free Encyclopaedia. http://en.wikipedia.org/wiki/Travelling_salesman_problem (accessed 22nd Jan. 2013, 11:10 UST). 


\section{Author' biography with Photo}

\section{Arindam Garai}

Me@ passionately love to mentor using innovation, mixing positive attitude and enthusiasm of a born leader and with strong desire to be a part of success in my chosen academic life.

Research Area: Fuzzy Set, Intuitionistic Fuzzy Optimization, Fuzzy and Intuitionistic Fuzzy Decision Making, Multiojective Optimization etc.

Mob.+91-9932890115

\section{Dr. Tapan Kumar Roy}

Me@ guide my students professionally as well as personally. Love studying as well as learning new progress made in my subject.

Research Area: Fuzzy and Intuitionistic Fuzzy set Theory, Inventory, Transportation, Reliability Optimization, Portfolio Optimization, Fuzzy and Stochastic Optimization, Multiojective Optimization etc.

Mob. $+91-9477419380$ 